\title{
Pelvic Infection, CTCAE
}

National Cancer Institute

\section{Source}

National Cancer Institute. Pelvic Infection, CT CAE. NCI Thesaurus. Code C143740.

A disorder characterized by an infectious process involving the pelvic cavity. 